\title{
Stiffness Matrix of Timoshenko Beam Element with Arbitrary Variable Section
}

\author{
Leiping $\mathrm{Xu}^{1-2, \mathrm{a}}$, Pengfei Hou ${ }^{1-2, b}$, Bing Han ${ }^{1, \mathrm{c}}$ \\ ${ }^{1}$ School of Civil Engineering, Southwest Jiaotong University, Chengdu 610031,China; \\ ${ }^{2}$ China Railway Major Bridge Reconnaissance \& Design Institute Co., Ltd.,China \\ axulp@brdi.com.cn, bhoupf1982@qq.com, chanbing0218_swjtu@163.com
}

Keywords: variable section; spatial beam element; stiffness matrix; equivalent nodal force; finite element

\begin{abstract}
Timoshenko beam with variable section is widely used for the sake of good mechanical behavior and economic benefit. In order to improve analytical accuracy, stiffness matrix of Timoshenko beam element with arbitrary section was founded. According to the relationship between geometrical deformation and element internal force, by integral of sectional curvature, shearing strain and axial strain, stiffness matrix of the Timoshenko beam element was derived. Then, analytical program was developed, which was proved exact in comparison with theoretical solution.
\end{abstract}

\section{Introduction}

Beams with variable section was widely used in large-span bridges which not only reduce the self-weight of structure significantly to improve spanning capacity, but also make full advantage of material. So accurate analysis of variable section beam makes great sense. In current studies, researchers usually adopt one of three methods to analyze beam element with variable section ${ }^{[1]}$. First method, beam element with variable section is considered as uniform beam based on average equivalent method ${ }^{[2-3]}$. Taking average of sections between two ends of the beam is one kind of average equivalent method. In the second method, stiffness matrix of beam element with variable section is founded by equilibrium equation ${ }^{[4]}$. In other studies, principle of minimum potential energy is adopted to establish the stiffness matrix of the beam element with variable section ${ }^{[5-8]}$.

Based on the current studies, the relationship between geometrical deformation and element internal force was established by integral of sectional curvature, shearing strain and axial strain. So the stiffness matrix of the Timoshenko beam element was derived directly from the relationship mentioned above. The shape function of element with arbitrary variable section was easily derived from the study, which could be applied to further studies. Then, program was developed based on the equation derived above. At last, numerical calculation about a cantilever beam structure was carried out, which prove that stiffness matrix of beam element with arbitrary variable section is correct.

\section{Calculation model}

In practical, sectional properties may vary irregularly along the element as shown in Fig.1.

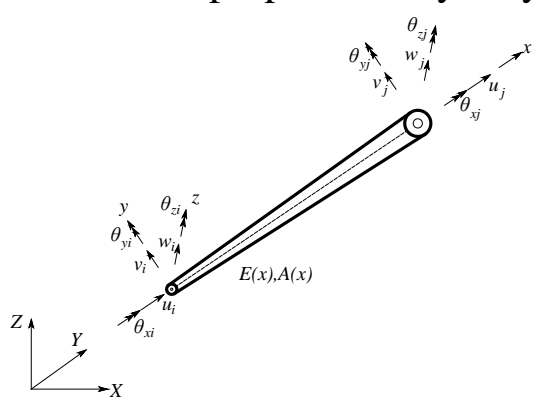

(a) nodal displacement

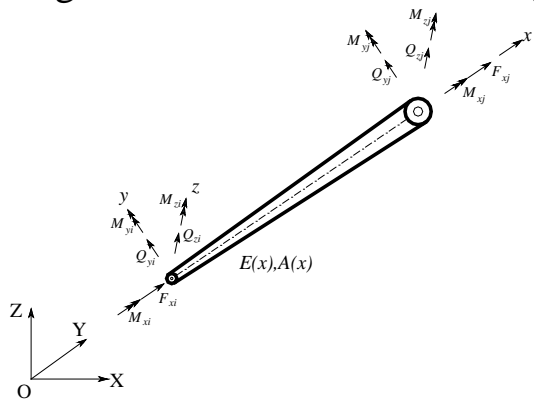

(b) elemental force

Fig.1 Spatial Timoshenko beam element with arbitrary variable section 
In Fig.1(a), $u, v$ and $w$ are displacements along $\mathrm{x}, \mathrm{y}$ and $\mathrm{z}$ respectively; $\theta_{x}, \theta_{y}, \theta_{z}$ are rotation angles around $\mathrm{x}, \mathrm{y}$ and $\mathrm{z}$ respectively; subscript $\mathrm{i}$ and $\mathrm{j}$ indicate node $\mathrm{i}$ and $\mathrm{j}$. In Fig.1(b), $M_{x}$ is torque around $\mathrm{x} ; M_{y}$ and $M_{z}$ are the moment around $\mathrm{y}$ and $\mathrm{z}$ respectively; $F_{x}$ is axial force; $F_{y}$ and $F_{z}$ are shear force along y and $\mathrm{z}$ respectively.

Variable functions of arbitrary section properties are assumed in the forms expressed in Eq.1,

$$
I(x)=I_{i} f_{I}(x) \quad A(x)=A_{i} f_{A}(x) \quad A_{s}(x)=A_{s i} f_{A_{s}}(x)
$$

where, $f_{I}(x) 、 f_{A}(x) 、 f_{A_{s}}(x)$ are distribution functions of inertial moment, axial area and shear area of beam element with arbitrary section respectively. Four variables are defined as shown in Eq.2 Eq.3.

$$
\begin{array}{cc}
L_{k 0}(x)=\int_{0}^{x} \frac{1}{f_{k}(u)} d u & L_{k 1}(x)=\int_{0}^{x} \frac{x}{f_{k}(u)} d u \\
L_{k 0 r}(x)=\int_{0}^{x} L_{k 0}(u) d u & L_{k 1 r}(x)=\int_{0}^{x} L_{k 1}(u) d u
\end{array}
$$

According to mechanical analysis of beam element as shown in Fig.1, sectional internal force of any position along the element can derived from formula as shown in Eq.4.

$$
\mathbf{F}_{\mathrm{I}}(x)=\mathbf{H}_{\mathrm{F}} \mathbf{F}_{\mathrm{I}}^{i}+\mathbf{F}_{\mathrm{I} p y}
$$

where, $\mathbf{F}_{\mathrm{I}}^{i}$ and $\mathbf{F}_{\mathrm{I}}(x)$ are respectively the section internal forces at section $\mathrm{i}$ and the section which is $\mathrm{x}$ away from the section $\mathrm{i} ; \mathbf{H}_{\mathrm{F}}$ is transfer matrix of section internal force. $\mathbf{F}_{\mathrm{I}}^{i}, \mathbf{F}_{\mathrm{I}}(x)$ and $\mathbf{H}_{\mathrm{F}}$ can expressed in Eq.5 Eq.7.

$$
\begin{gathered}
\mathbf{F}_{\mathrm{I}}(x)=\left[\begin{array}{cccccc}
N(x) & Q_{\mathrm{y}}(x) & Q_{\mathrm{z}}(x) & T(x) & M_{\mathrm{y}}(x) & M_{\mathrm{z}}(x)
\end{array}\right]^{\mathrm{T}} \\
\mathbf{F}_{\mathrm{I}}^{i}=\left[\begin{array}{llllll}
N_{i} & Q_{\mathrm{y} i} & Q_{\mathrm{zi}} & T_{i} & M_{\mathrm{y} i} & M_{\mathrm{zi}}
\end{array}\right]^{\mathrm{T}} \\
\mathbf{H}_{\mathrm{F}}=\left[\begin{array}{cccccc}
-1 & 0 & 0 & 0 & 0 & 0 \\
0 & -1 & 0 & 0 & 0 & 0 \\
0 & 0 & -1 & 0 & 0 & 0 \\
0 & 0 & 0 & -1 & 0 & 0 \\
0 & 0 & -x & 0 & -1 & 0 \\
0 & x & 0 & 0 & 0 & -1
\end{array}\right]^{\mathrm{T}}
\end{gathered}
$$

The stiffness matrix of beam element in plane xoy can be first derived, the equivalent value in plane xoz can be similarly derived. Subscript y in variables defined above facilitate derivation. Then section internal force vector in plane xoy can be expressed as Eq.8

$$
\mathbf{F}_{\mathrm{I} y}(x)=\mathbf{H}_{\mathrm{Fy}}(x) \mathbf{F}_{\mathrm{Iy}}^{i}+\mathbf{F}_{\mathrm{I} p y}
$$

\section{Derivation of stiffness matrix}

\section{Axial and torsional stiffness}

Axial strain and twist ratio of beam element with variable section is not constant, the axial displacement can be derived by integral as shown in Eq.9,

$$
N_{i}=\frac{E A_{i}\left(u_{i}-u_{j}\right)}{L_{A 0}(l)}
$$

where, $N_{i}$ is the axial force in node $\mathrm{i}$.

Axial force at section $\mathrm{j}$ could be derived from $N_{i}$ based on the static equilibrium equation. Likewise, torque is derived from formula as shown in Eq.10.

$$
T_{i}=\frac{G I_{x i}\left(\theta_{x i}-\theta_{x j}\right)}{L_{I_{x} 0}(l)}
$$




\section{Bending and shearing stiffness}

Total vertical displacement includes two parts as shown in Eq.11,

$$
v=v_{b y}+v_{s y}
$$

where, $v_{b y}$ is the displacement induced by bending moment, $v_{s y}$ is the displacement induced by shearing force.

Firstly, taking vector $\boldsymbol{\delta}_{y}$ as section response as shown in Eq.12,

$$
\boldsymbol{\delta}_{y}(x)=\left[\begin{array}{ll}
v_{y}(x) & \theta(x)
\end{array}\right]^{\mathrm{T}}
$$

According to mechanics of materials, relationship between displacements and section internal force was established as shown in Eq.13,

$$
\mathbf{F}_{I y}^{i}=\mathbf{H}_{1 y} \mathbf{H}_{2 y} \mathbf{d}_{y}^{e}
$$

where, $\quad \mathbf{F}_{l y}^{i}=\left[\begin{array}{ll}Q_{y i} & M_{z i}\end{array}\right]^{\mathrm{T}}, \mathbf{d}_{y}^{e}=\left[\begin{array}{llll}v_{y i} & \theta_{z i} & v_{y j} & \theta_{z j}\end{array}\right]^{\mathrm{T}}, \mathbf{H}_{1 y}=\left[\begin{array}{l}\mathbf{N}_{d y}(l)\end{array}\right]^{-1}$

$$
\begin{gathered}
\mathbf{H}_{2 y}=\left[\begin{array}{cccc}
-1 & -l & 1 & 0 \\
0 & -1 & 0 & 1
\end{array}\right] \\
\mathbf{N}_{d y}(x)=\frac{1}{E I_{z i}}\left[\begin{array}{cc}
L_{I_{z} 1 r}(x)-\frac{l^{2} b_{y}}{12} L_{A_{s y} 0}(x) & -L_{I_{z} 0 r}(x) \\
L_{I_{z} 1}(x) & -L_{I_{z} 0}(x)
\end{array}\right]
\end{gathered}
$$

Stiffness matrix was further derived from Eq.9, Eq.10 and Eq.13.

For uniform beam element, variable functions described in Eq.1 equal 1. Parameters defined in Eq.2 Eq.3 will be simplified as shown in Eq.16 Eq.17.

$$
\begin{gathered}
L_{k 0}(x)=x \quad L_{k 1}(x)=\frac{1}{2} x^{2} \\
L_{k 0 r}(x)=\frac{1}{2} x^{2} \quad L_{k 1 r}(x)=\frac{1}{6} x^{3}
\end{gathered}
$$

Then, stiffness matrix of uniform beam element was derived.

\section{Example analysis}

A FEA program was developed based on the equation derived above. A cantilever beam as shown in Fig.2 is selected as the example object. The beam height varies linearly along the axes. Load and dimension of the structure are listed in Table1.

Table 1. Calculation parameter

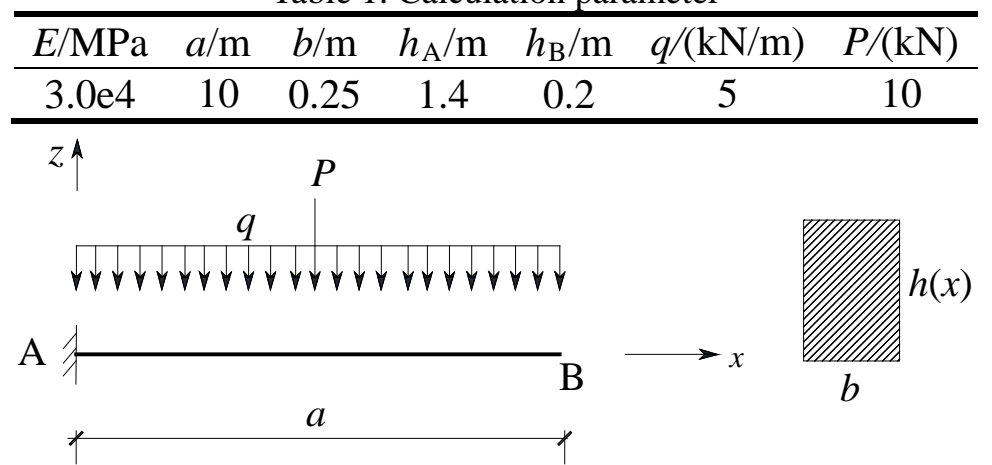

Fig.2. Cantilever beam with rectangular section

According to structural mechanics, vertical displacement of section $\mathrm{B} v_{\mathrm{B}}=-9.7838 \mathrm{~mm}$, and sectional rotation angle $\theta_{\mathrm{B}}=0.001798 \mathrm{rad}$. Displacement and rotation angle in section $\mathrm{B}$ calculated from different models are showed in Fig.3. Lateral axis shows number of equivalent section applied to simulate the structure by average equivalent method. 


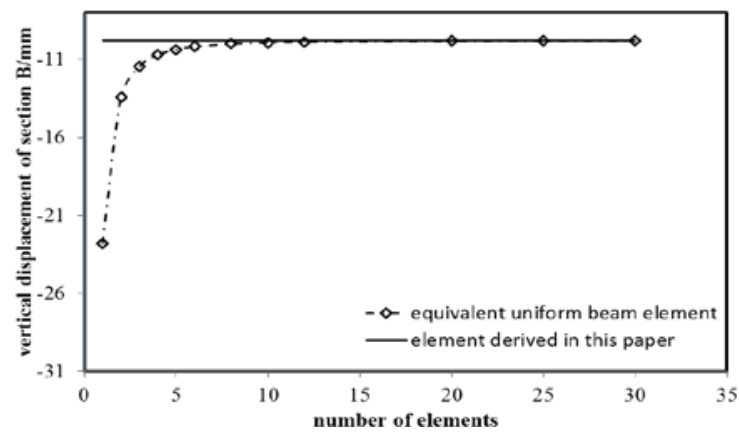

a Nodal displacement

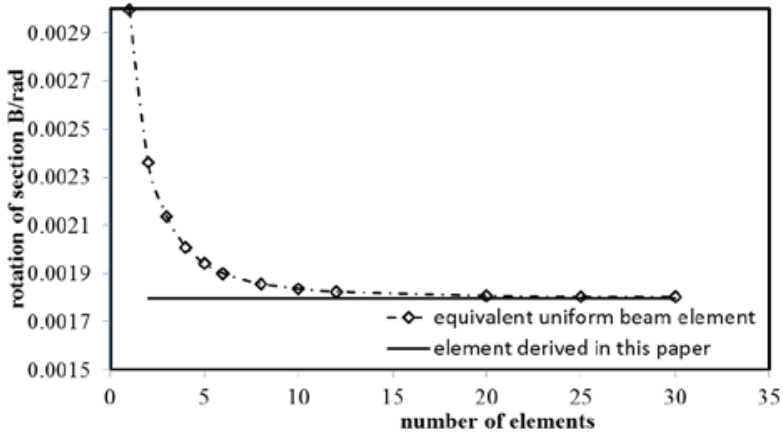

b Rotation angle

Fig.3. Response of section B calculated from different models

As shown in Fig.3, response calculated from model with only one element derived in this paper is exact compared to theoretical solution. In the contrary, more elements are needed to get exact solution for models based on average equivalent method.

\section{Conclusion}

1) Stiffness matrix of Timoshenko beam element with variable section was derived by geometrical analysis and element equilibrium condition.

2) Based on the theoretical study, the calculation program was developed. Numerical calculation was carried on a cantilever beam structure which shows that the Timoshenko beam element with variable section is correct.

\section{References}

[1] CHUAN Guang-hong,CHEN YI-yi, TONG Gen-shu. Element stiffness matrix for Timoshenko beam with variable cross-section[J]. Chinese Journal of Computational Mechanics, 2014,31(2): 265-272.

[2] LIU Yue, WANG Li-ping. Simplification of a spatial beam with variable cross section based on the stiffness matrix[J]. Tsinghua Univ(Sci\&Tech),2008,48(11):1915-1918.

[3] Du X X, Peng P, Luo F X. Calculation of any element with tapered beam member based on the method of equivalent moment of inertia[J]. Advanced Matedials Research,2011,243-249:650-655.

[4] MA Xi ling, XIAO Zheng hua. The accurate equation of element stiffness matrix for variable cross section bars[J].Journal of Xin Jiang Institute of Technology,2000,21(1):1-5.

[5] Wang Xiao-chen, PU Jun-ping. The finite element analysis of non-uniform beams[J]. Journal of Zhejiang University of Technology, 2008,36(3):311-315.

[6] ZHAO Bin,Wang Zheng-zhong. Study on a mechanics model of wedge-shaped elements[J]. Research on iron\&Steel,2002,30(5):28-30.

[7] Fan Sheng gang, Shu Gan-ping, Lv Zhi-tao. Stability analysis of steel beams with tapered sections[J] Special Structures,2002,19(4):36-40.

[8] ZHANG Yuan-hai. A nonuniform beam finite element in engineering structure analysis[J]. Journal of LANZHOU railway university(Natural sciences) ,2000,19(4),59:62. 